\title{
A framework for manufacturing execution system deployment in an advanced additive manufacturing process
}

\author{
Gianluca D'Antonio* \\ Politecnico di Torino, \\ C.so Duca degli Abruzzi 24, 10129 Torino, Italy \\ Email: gianluca.dantonio@polito.it \\ *Corresponding author
}

\section{Frédéric Segonds and Floriane Laverne}

Ècole Nationale Superieure d'Arts et Métiers, 151, bd de l'Hôpital, 75013 Paris, France

Email: frederic.segonds@ensam.eu

Email: floriane.laverne@ensam.eu

\section{Joel Sauza Bedolla and Paolo Chiabert}

Politecnico di Torino,

C.so Duca degli Abruzzi 24,

10129 Torino, Italy

Email: joel.sauza@polito.it

Email: paolo.chiabert@polito.it

\begin{abstract}
The deployment of additive manufacturing (AM) processes had a rapid and broad increase in the last years, and the same trend is expected to hold in the near future. A way to better exploit the advantages of such technology is the use of appropriate information tools. However, today there is a lack of software applications devoted to this innovative manufacturing process. To overcome this issue, in the present work the application of manufacturing execution systems (MES), a tool commonly used in traditional manufacturing processes, is extended to AM. Furthermore, a framework for the deployment of shop-floor data, acquired through a monitoring system, in the design phase is presented: hence, MES should cooperate with design for additive manufacturing (DFAM), a set of methods and tools helpful to design a product and its manufacturing process taking into account AM specificities from the early design stages. In order to better understand the advantages of such cooperation, a case study for a proof of concept has been developed: the obtained results are promising, thus an online implementation would be recommended.
\end{abstract}

Keywords: design for additive manufacturing; DFAM; additive manufacturing process; information systems; monitoring systems; manufacturing execution system; MES. 
Reference to this paper should be made as follows: D'Antonio, G., Segonds, F., Laverne, F., Sauza Bedolla, J. and Chiabert, P. (2017) 'A framework for manufacturing execution system deployment in an advanced additive manufacturing process', Int. J. Product Lifecycle Management, Vol. 10, No. 1, pp.1-19.

Biographical notes: Gianluca D'Antonio is a Post-Doc Fellow in Production Systems at Politecnico di Torino (Italy). His research areas cover the automation systems, the development of mathematical techniques for real-time data analysis and their integration into manufacturing execution systems to support the implementation of lean manufacturing techniques.

Frédéric Segonds is an Assistant Professor at Arts etMétiersParisTech and member of the product design and innovation laboratory (LCPI). His research interests focus on early stages of design collaboration, collaborative design and design for/with additive manufacturing (DFAM/DWAM). It includes the integration of stakeholder's core competences into early stages of design, and provides assistive methodologies and tools to support the product design.

Floriane Laverne holds a $\mathrm{PhD}$ in Industrial Engineering from the Arts et Metiers ParisTech School of Engineering in Paris, France and is member of the Product Design and Innovation Laboratory (LCPI). Her research interests focus on design with and design for additive manufacturing during early design stages. This includes developing methodologies and tools to help designer better use additive manufacturing knowledge.

Joel Sauza Bedolla is a Post-Doctoral Research Fellow at Politecnico di Torino (Italy). He received his $\mathrm{PhD}$ in Production Systems from Politecnico di Torino in 2013. He conducted research on PLM by studying product definition and verification processes according to the new ISO/TR-14638 Masterplan. He is also been responsible of the introduction of PLM-based exercise in the bachelor program of Automotive Engineering at Politecnico di Torino.

Paolo Chiabert is an Associate Professor at Politecnico di Torino (Italy). He graduated in Electronic Engineering, Politecnico di Torino (1993), IBM consultant (1994), a researcher in design and methods of industrial engineering at the Politecnico di Torino (1995-2003), an Associate Professor in Technology and Manufacturing Systems (2004). According to the product lifecycle management perspective, the research interests range from design methods, to manufacturing technologies, to production planning and control, to quality management. Since 1997 Italian expert in the ISO Technical Committee 213 - geometrical product specifications and verification, he is member of the GPS Technical commission at UNI.

This paper is a revised and expanded version of a paper entitled 'A proposal of manufacturing execution system integration in design for additive manufacturing' presented at The 12th International Conference on PLM, Doha, Qatar, 19-21 October 2015. 


\section{Introduction}

In the ASTM (2012) standard, additive manufacturing (AM) is defined as "the process of joining materials to make objects from 3D model data, usually layer upon layer, as opposed to subtractive manufacturing methodologies, such as traditional machining". Several synonyms are also defined for AM: additive fabrication, additive processes, additive techniques, additive layer manufacturing, layered manufacturing, rapid manufacturing and freeform fabrication. To perform an additive fabrication process, the model data of the object to be produced is decomposed into a number of 2D cross sections and a file is created: common standards for such file are STL, VRML or the recently introduced $3 \mathrm{MF}$. Then, the file is sent to the AM machine, which adds material layer by layer to produce the physical object.

$\mathrm{AM}$ is emerging as an important manufacturing process and a key technology for enabling innovative product development (Laverne et al., 2015). Nevertheless, to support and promote this technology and its advantages, appropriate information tools are necessary. Currently there is a lack of software applications devoted to this innovative manufacturing process. In particular, few work has been done in the deployment of real-time monitoring systems to improve the quality of the product and the stability of the process. However, the problem of machine monitoring has industrial relevance. EOS has deposited a patent (Perret and Philippi, 2014) for the automatic calibration of a machine through a scanner; the MIT deposited a patent (Perez et al., 2015) for an AM process control methodology including a camera to acquire images of the object being manufactured. In traditional manufacturing processes, monitoring and control systems are integrated into manufacturing execution systems (MES). Nonetheless, in literature no applications of MES exist in the field of AM. Thus, the scientific contribution of this paper is to extend the state of the art providing a framework for the deployment of new information tools for AM; a case study for the monitoring of surfaces created by 3D printers will be introduced to validate the framework. Beside quality monitoring, the information extracted by such monitoring system can be deployed during the design phases for product or process adjustments. Hence, the integration between MES and design for additive manufacturing (DFAM), a set of methods and tools helpful to design a product and its manufacturing process taking into account AM specificities from the early design stages (Laverne et al., 2015), will be also discussed.

The remainder of the paper is organised as follows: in Section 2 we briefly review AM technologies and methodologies to support design. In Section 3 we introduce MES and explain its possible deployment in AM. In Section 4 the developed case study is presented. The role of MES is discussed in Section 5 and a framework for its cooperation with DFAM is presented in Section 6. Finally, conclusive remarks are provided in Section 7.

\section{Additive manufacturing}

\subsection{AM processes}

Several AM processes are currently available; seven process categories are defined in the ASTM standard. The choice of the fabrication process is strictly tied to the deployed material: polymers, metals, ceramics and organic materials are among the main ones 
(Doubrovski et al., 2011). Material extrusion is one of the most deployed methodologies: a thermoplastic material is heated over its glass transition temperature and extruded through a nozzle in a controlled manner. The extruded material is used to print $2 \mathrm{D}$ sections successively, one on top of another, until the object is complete. ABS and PLA are the most common thermoplastic polymers in material extrusion, because of their relatively low glass transition temperatures (Mellor et al., 2014; Rao et al., 2015). Recently, an innovative technique, named CLIP, has been developed and patented: a continuous liquid interface is used to build 3D objects; it is much faster than 'traditional' additive techniques (Tumbleston et al., 2015). Metal AM techniques are mainly based on powder; the mostly used materials are steels, pure titanium and titanium alloys, aluminium casting alloys; this list of metals and alloys is continuously growing as new processes are developed (NIST, 2013). Nevertheless, currently no process is able to create net shape parts, and a post-processing operation is necessary (for example, to remove supports or to finish the surface). This kind of operations can lead to some deformations and, sometimes, to destruction of the AM part. Consequently, post-processing operations can be a source of functional problems for the part: dimensional, shape, roughness errors, etc. Thus, a stand-alone AM implementation is not yet feasible, and the integration among production processes is necessary (Mellor et al., 2014).

AM processes allow an extraordinary design freedom: this advantage makes feasible shape complexity and geometry customisation levels that are not reachable with traditional manufacturing technologies (Vayre et al., 2012). Furthermore, material waste is reduced, since structural parts without functionalities for the user can be unnecessary (NIST, 2013). Time-to-market is shortened with respect to traditional processes, both because the design can be quicker and because additive fabrication totally occurs in a single place (while traditional processes can take place in different locations). This makes feasible a just-in-time production approach, as well as a reduction in transportation issues, cost and energy consumption (Vayre et al., 2012).

Figure 1 Comparison among the timelines for industrial revolutions, traditional manufacturing and AM technologies (see online version for colours)

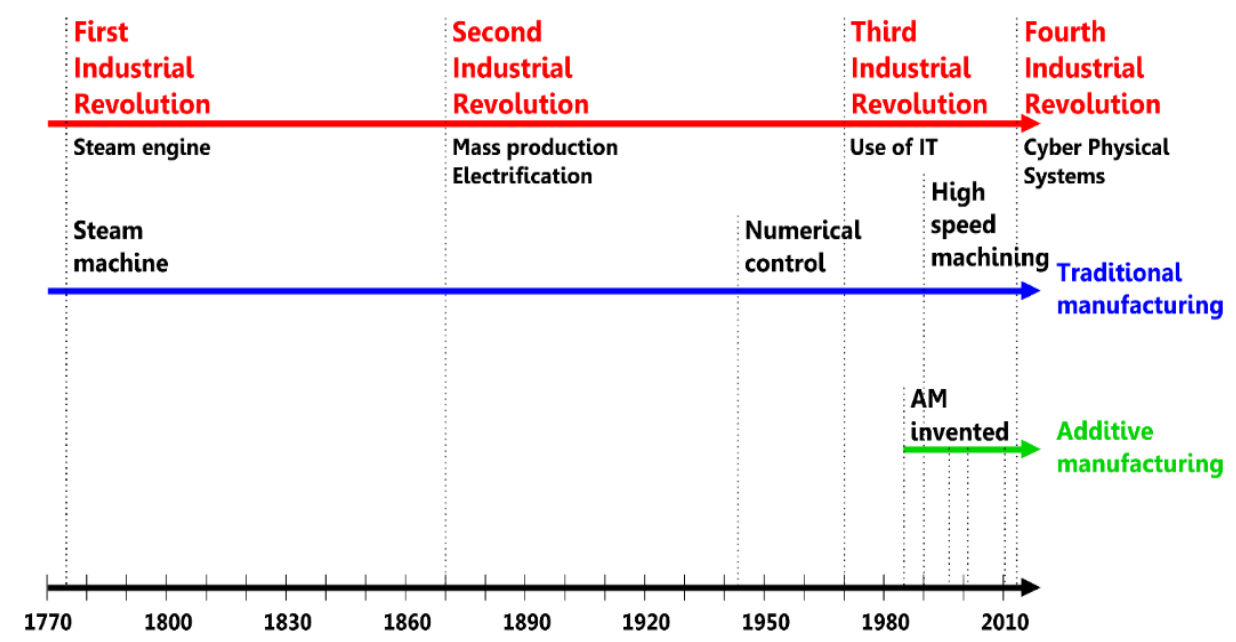


Figure 2 Focus on the timeline for AM development (see online version for colours)

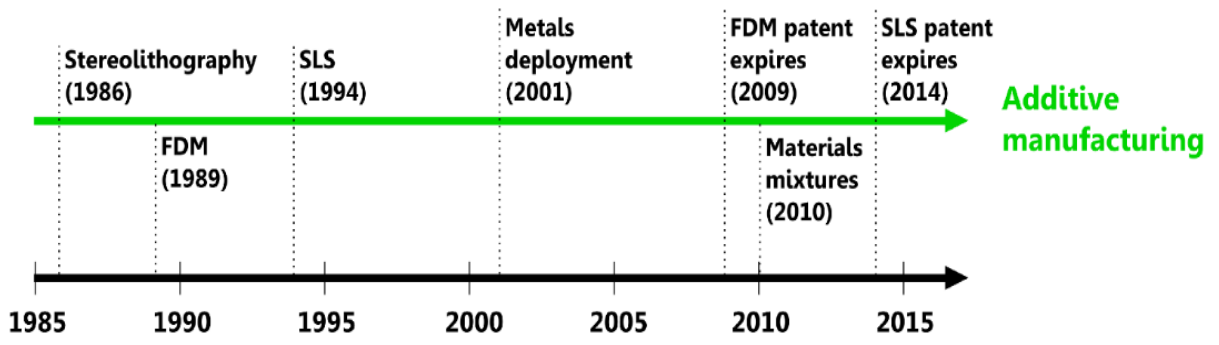

AM processes are quite new, compared to traditional manufacturing processes: in Figure 1, a comparison between the timelines for industrial revolutions and the development of traditional and AM techniques is shown; however, AM development has been very quick and rich of milestones (see Figure 2). This class of process is still rapidly changing, and new applications arise as new materials become available. This rapid growth is also due to the support of the technological tools that led to the third and the fourth industrial revolutions. Currently, AM technology is mainly deployed in aerospace, automotive and biomedical devices manufacturing. The high customisation level allows to profitably use freeform fabrication in personalised products and in the production of small lots (Atzeni and Salmi, 2012; Mellor et al., 2014). Beyond end-user products, an indirect usage of AM is also feasible, for example to develop and produce tools for conventional machines as well as for reverse engineering of components which are out of production or under maintenance (Vayre et al., 2012).

The AM market is significantly growing in every manufacturing sector: according to the last report by Wohlers Associates (2014), the global AM market in 2013 was $\$ 3.07$ billion (corresponding to Euro 2.8 billion, given a change rate equal to 1 dollar $=1.11$ euro); the yearly increase, with respect to the 2012 is $35 \%$.

\subsection{AM methodologies}

The hype cycle presented in Figure 3 (Basiliere and Shanler, 2014), envisages that in the next five to ten years AM will be deployed in several different production fields such as bioprinting systems, oil and gas, and medical devices. As shown in the previous section, a huge research effort has been made to develop new materials and fabrication techniques; conversely, for a long time little investigation has been performed on the methods for designers. Yet, the design has a remarkable impact on the downstream phases, e.g., production, distribution, utilisation and disposal. The DFAM methodologies are now a major issue to exploit in an appropriate way the potential of AM technologies for product development (Laverne et al., 2015). Furthermore, digital fabrication and on-demand production dramatically changed the manufacturing paradigms: a customer can look for a product in a digital catalogue, customise it and send the resulting file to a small firm to fabricate it (Doubrovski et al., 2011). AM allows to produce huge quantities as well as small volumes of a product, with little or no stock; however, this technology is not yet used for the production of large products lots, because of economic reasons (Atzeni and Salmi, 2012).

To manufacture high-quality products, the properties of the material must be well known; these properties can strongly vary according to the production parameters, such 
as the orientation of the part in the 3D printer, the build speed and the tool path. Thus, the deployment of a consistent and structured design approach is mandatory. In traditional processes, design for manufacturing (DFM) practice is deployed to eliminate production issues, and minimise manufacturing, assembly and logistics costs (Boothroyd et al., 1994). However, additive processes have different constraints and DFM cannot be used as it is; it must be re-thought to take into account the unique capabilities of AM, in order to fully exploit the advantages of such technology and consider its limits from the early design stage (Ponche et al., 2012). In particular, new design tools are necessary to define and explore product shape and properties, new materials, new efficient manufacturing processes, and to assess lifecycle costs (Huang et al., 2015).

Figure 3 Hype cycle for AM technology (see online version for colours)

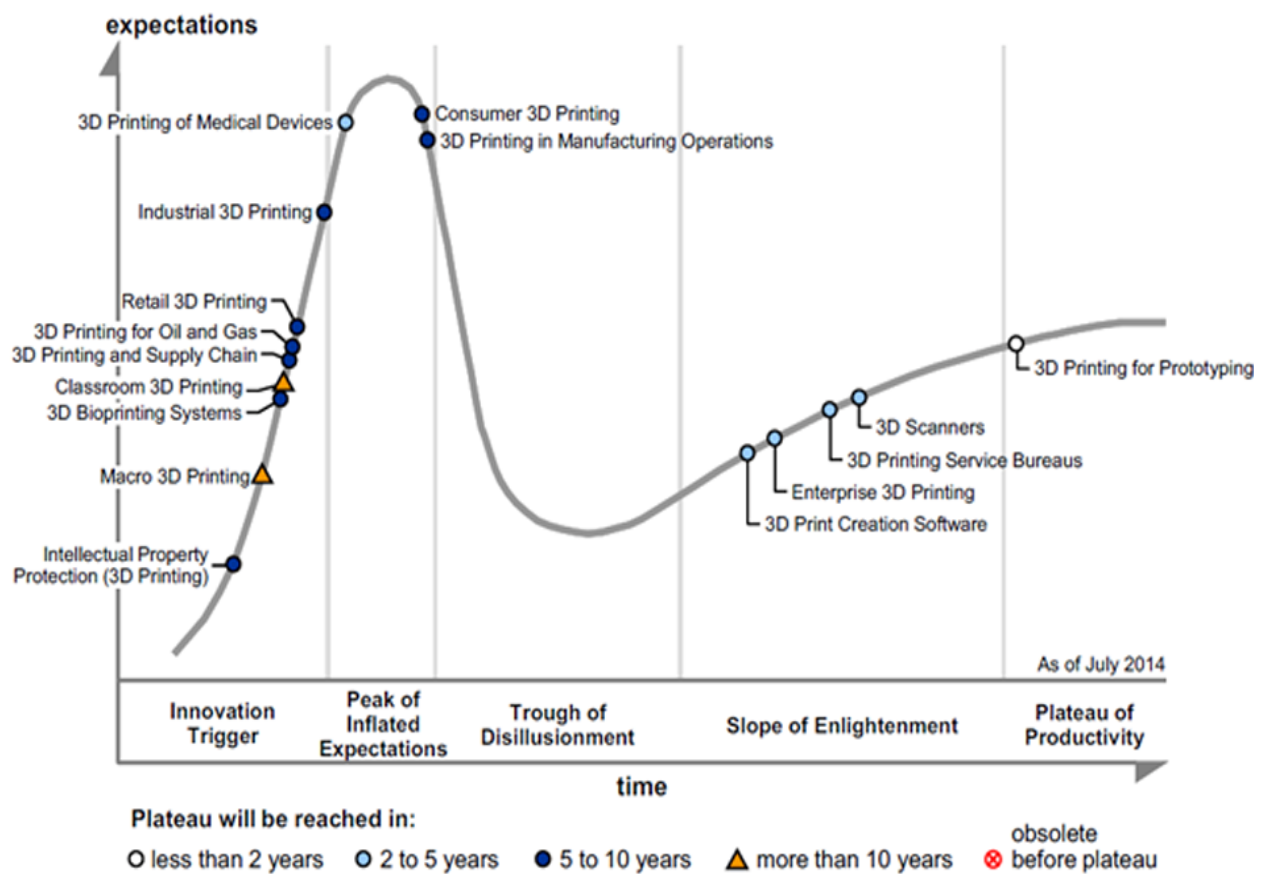

Source: Basiliere and Shanler (2014)

Figure 4 Design for DFAM methodology

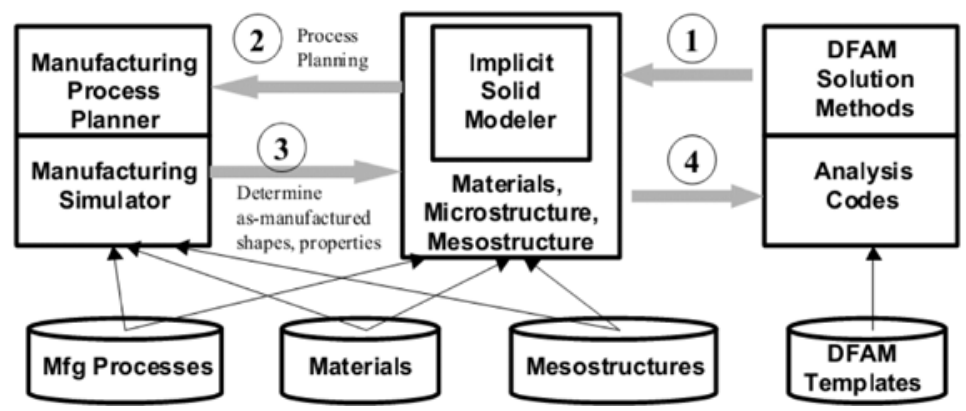

Source: Rosen (2007) 
DFAM is a set of methods and tools helpful to design a product and its manufacturing process taking into account AM specificities from the early design stages: DFAM allows to determine an optimised process planning from the functional specifications (Ponche et al., 2014). Mançanares et al. (2015) developed a method to select the best AM process for a part, in order to best satisfy the target. Rosen (2007) defined DFAM as the synthesis of shapes, sizes, geometric mesostructures, material compositions and microstructures to best utilise manufacturing process capabilities to achieve desired performance and other life-cycle objectives. He also defined the DFAM structure shown in Figure 4. Design is represented by the right-left flow: functional requirements are transformed into properties and an appropriate and realistic geometry; a process planning is performed to formulate a potential manufacturing process. On the left-right flow, the designed product and its fabrication are simulated to determine how well the original requirements are satisfied. Another structure for DFAM is formulated by Ponche et al. (2014): their methodology - shown in Figure 5 - is organised in three steps: determination of part orientation into the machine; topological optimisation of the part; optimisation of the manufacturing paths. This methodology allows to take into account the characteristics and constraints of the chosen AM process from the early stage of design.

Figure 5 Design for DFAM methodology (see online version for colours)

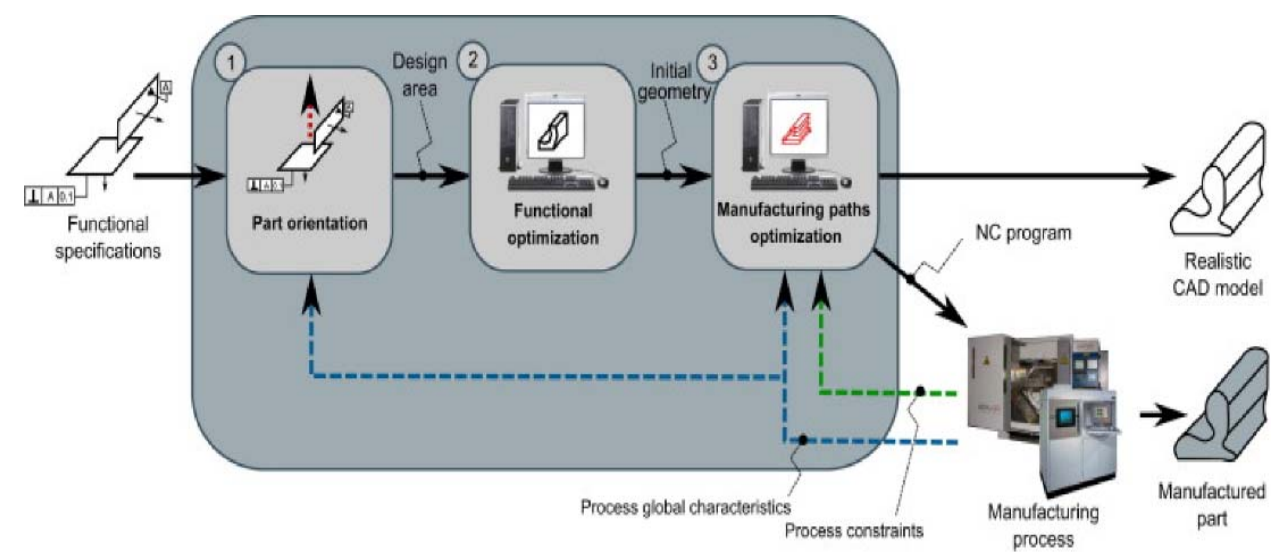

Source: Ponche et al. (2012)

Thus, different definitions for DFAM are given in literature. The two models provided above mainly focus on the design of a process for producing single parts. Nonetheless, the approach can be extended to multi-part processes. For example, Zhang et al. (2015) developed a genetic algorithm to identify the best orientation in case of multi-part production, to minimise the cost of the process and the requested time.

Beside design tools, in this work we also focus on information systems to collect and analyse data from the additive machine. This functionality is typically integrated into MES, which are introduced in the following section. 


\section{Manufacturing execution systems}

MES can be defined as a group of information tools that enable information exchange between the organisational level of a company, which is commonly supported by an ERP, and the control systems for the shop-floor, usually consisting in several, different, very customised software applications (Meyer et al., 2009). Thus, MES is a strong support to intelligent manufacturing.

A MES has two principal purposes. First, the system is in charge of evaluating the optimal production planning by taking into account the constraints of the process, such as processing and setup times, workstations capacity, requirements, necessities and targets given by the organisational level. The MES also has to manage and allocate resources such as the staff and the material necessary for the manufacturing process.

The second aim of a MES is to manage the bottom-up data flow: recently, the development of low-cost, small, easily available sensors led to increased diffusion of monitoring systems to assess product quality and process performance, and to support the improvement of a production process. The role of MES is to collect the shop-floor information, analyse it through proper mathematical techniques, and provide an exhaustive description of the current state of the process. Possibly, the analysis should be performed in real-time, in order to make decisions to control the process as fast as possible. Such analyses can be aimed to quality monitoring, process management and optimisation, product traceability, or predict and prevent maintenance approach. A synthesis of MES integration with other information tools and the two data flows is given in Figure 6. Examples of real-time monitoring systems integrated in MES are provided in some papers (Arica and Powell, 2014; Snatkin et al., 2013; Zhong et al., 2013).

Figure 6 MES integration with ERP and the information tools used at the shop-floor level (see online version for colours)

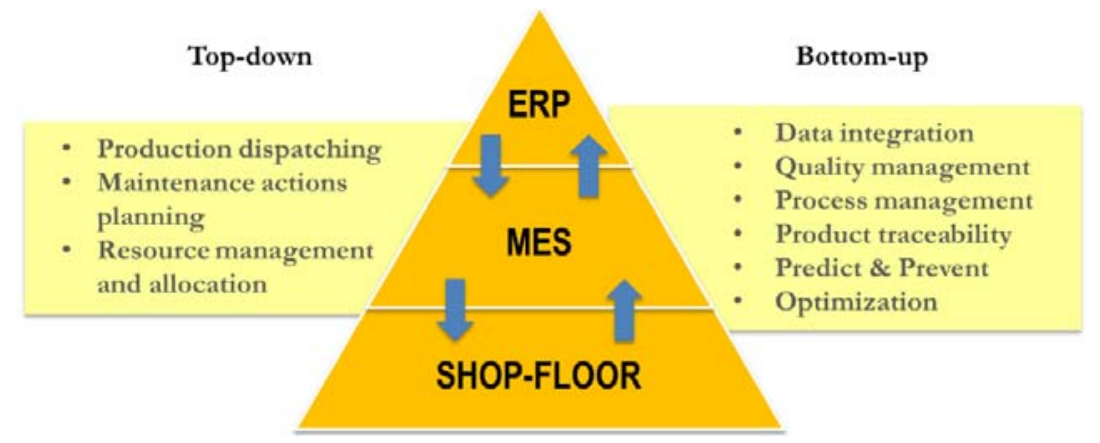

MES for AM. At the state of the art, there is no application of MES in the field of AM. In literature, some predictive models based on the values of machine parameters have been developed. Vijayaraghavan et al. (2014) formulated a model to predict the wear strength of a part, based on layer thickness, orientation, air gap, raster angle and width. Byun and Lee (2006) developed a decision making strategy for part orientation based on surface quality, building time and part cost. Sood et al. (2012a, 2012b) used air gap, raster angle and raster width to predict the wear rate and the compressive resistance of a part. Boschetto and Bottini (2014) developed a model to predict dimensional deviations of fabricated parts as a function of the process parameters. However, machine 
parameters are not sufficient to predict possible anomalies and failures; hence, the use of sensor-based monitoring systems is necessary. Rao et al. (2015) measured vibrations and temperatures to optimise process conditions, in order to obtain the best surface roughness and to real-time detect possible drifts. Bukkapatnam and Clark (2006) used a set of accelerometers to trace in real-time variations in process dynamics and to early detect possible anomalies. Dimensional and geometrical measurement and control of the manufactured parts also play a key role. Faes et al. (2014) deployed an optical sensor to measure layer width and height and to control the geometrical error in the $z$ direction (perpendicular to slicing direction, traditionally vertical).

Process parameters can be used to estimate part quality in an indirect way. However, direct measurements usually lead to more reliable results (Abellan-Nebot and Subiron, 2010). Two kinds of technologies can be used to directly evaluate the quality of the produced geometry. Contact measurements through a probe are not currently feasible in $\mathrm{AM}$, since the part is fixed in the manufacturing environment, and the room for a moving probe is very scarce; this technology can be used for offline measurements, after removing the part from the machine. For example, the object can be measured onto a coordinate measuring machine (CMM); this process leads to high quality measurements, but requires a huge amount of time, both for the measurement and for the creation of the measuring path. An alternative technology is given by non-contact measurements. Several different tools can be used to measure an object in a few seconds, according to the available room, the dimension of the object to be measured and the desired accuracy of the output. For example, optical measurements can be performed and different technologies can be chosen (such as time of flight or stereoscopic vision).

At the state of the art, the deployment of real-time monitoring systems in AM is restricted because of the lack of proper smart sensors. This is mainly due to two factors. The first one is the reduced access to the build chamber: in high-performance additive machines, the printing environment is closed to keep constant the temperature, and cannot be opened. On the other side, the temperatures are too high for a common measuring device. The second factor is the need for intensive computing power: due to the very small time scale at which additive phenomena occur, fast and reliable in-situ measurements and analysis must be performed.

Given the importance of MES role in AM combined with a monitoring system, in the next sections the core part of this research work is introduced: a case study for offline optical measurement of an additive manufactured part and its deployment into a MES is provided. Further, a theoretical framework is presented for the cooperation between MES and DFAM: this allows the design to have a feedback from the events occurring at the shop-floor.

\section{Experimental case study}

\subsection{Description of the process}

Before the development of a customised industrial solution, it is important to validate our methodology (presented in the next section) on a product. We carry tests on a set of toys representing hollow ducks fabricated through a BFB 3D Touch machine, an additive machine based on the fused deposition modelling technique. Although a toy, the geometry of this part is not trivial at all, from the building process perspective: the 
surface exhibits different curvatures with variable radii. The dimensions of the parts (as designed) are $80.6 \mathrm{~mm}$ (length), $45.6 \mathrm{~mm}$ (width) and $51.5 \mathrm{~mm}$ (height). The objects have been produced both in PLA and in ABS. After the production of some parts, a non-detected issue in axes calibration arose, leading to surface defects of the final products. In particular, the part shown in Figure 7 exhibits a hole which width is $1.5 \mathrm{~mm}$.

Figure 7 The part used as a case study, which exhibits a fabrication defect (see online version for colours)

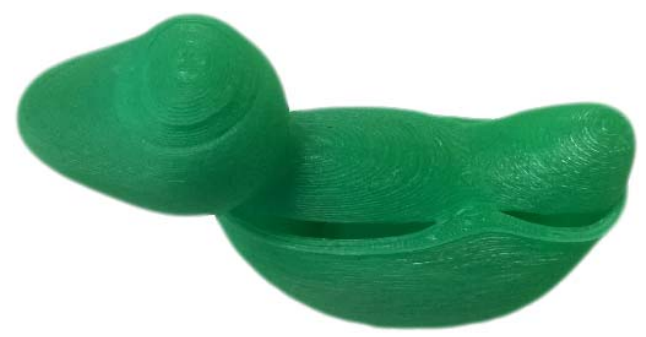

\subsection{Monitoring and control system}

Due to the lack of sensors discussed in the previous section, in this study offline laboratory measurements have been performed: the whole profile of the defective part has been measured after the fabrication. The surface has been scanned through a GOM Atos machine; the distance between adjacent measured points is $0.6 \mathrm{~mm}$. Then, the real-time behaviour of the technique for measurement analysis has been simulated: the algorithm was supposed to be run at every $2 \mathrm{~mm}$ material deposition thickness. The algorithm has been implemented in Matlab, and consists in two parts: in the first one, the presence of possible discontinuities in the surface of the part is detected. In the second part, the scanned point's grid is compared with the nominal points cloud to evaluate the adherence to the expected output. In the following, a pseudo-code of the two algorithms is provided (also see Figure 8):

1 Test for part integrity:

a Identify the points without close neighbours: for each scanned point, evaluate whether other surface points were found in a square centred in the point with edge size equal to $2 \mathrm{~mm}$.

b Identify the boundary points: for each scanned point, evaluate whether there exist some points that can be considered neighbours or not. For each direction, a $0.5 \times 20 \mathrm{~mm}$ rectangle is considered: if no points are detected into this area, the point is tagged as boundary point.

c Identify the points without close neighbours that are not boundary points, i.e., the points found in step 1a which do not respect the criteria in item $1 \mathrm{~b}$.

d The points identified at step 1c are tagged as risky points: the lack of close neighbours and the presence of other, far, points with a similar $x$ or $z$ coordinate could be due to a discontinuity in the surface of the object. 
2 Alignment to the nominal geometry:

a The CAD file for the nominal geometry is transformed into a points cloud.

b The scanned points are roughly aligned to the nominal geometry: three translations are performed to align the two centres of mass, as well as rotations to align the axes directions.

c The iterative closest point (ICP) algorithm (Besl and McKay, 1992) is used to best align the measured points cloud to the nominal model, by minimising the distances between the two surfaces. The repositioning performed at item $2 b$ is used as initial condition for the ICP, and allows to reduce computational time.

d For each scanned point the distances from the nominal surface are measured.

Figure 8 Pseudo-code of the algorithms for measurements analysis (see online version for colours)

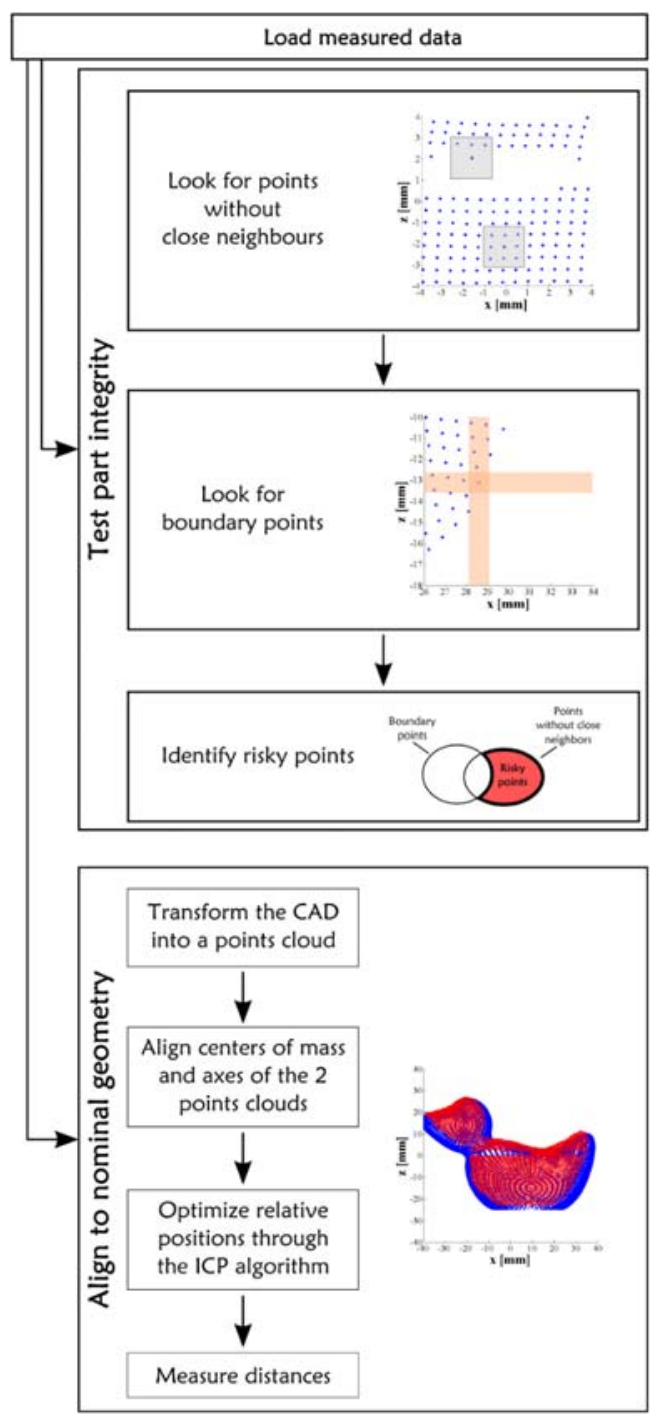


The values for the square and the rectangle sizes have been chosen by combining:

1 the properties of the measurement system, having a sampling step equal to $0.6 \mathrm{~mm}$

2 the geometrical features of the part: the distance between the end of the neck and the beginning of the tail is nearly $25 \mathrm{~mm}$; in case the size of the rectangle is close or greater than this value, some points on the neck and on the tail would be wrongly tagged as risky.

\subsection{Results}

In Figure 9 some snapshots from the simulation for the integrity test algorithm are shown. Points on the surface of the duck are plotted in cyan; black points are the boundary points; red dots represent the risky points, i.e., the points which do not have close neighbours, but cannot be considered as boundary points (identified at step 1c). Thus, red points would not exist if the surface of the object does not exhibit discontinuities. In the example, 4,612 points are scanned on the surface of the object; among them, 115 are considered risky.

Figure 9 Results of the simulation for the integrity test algorithm (see online version for colours)
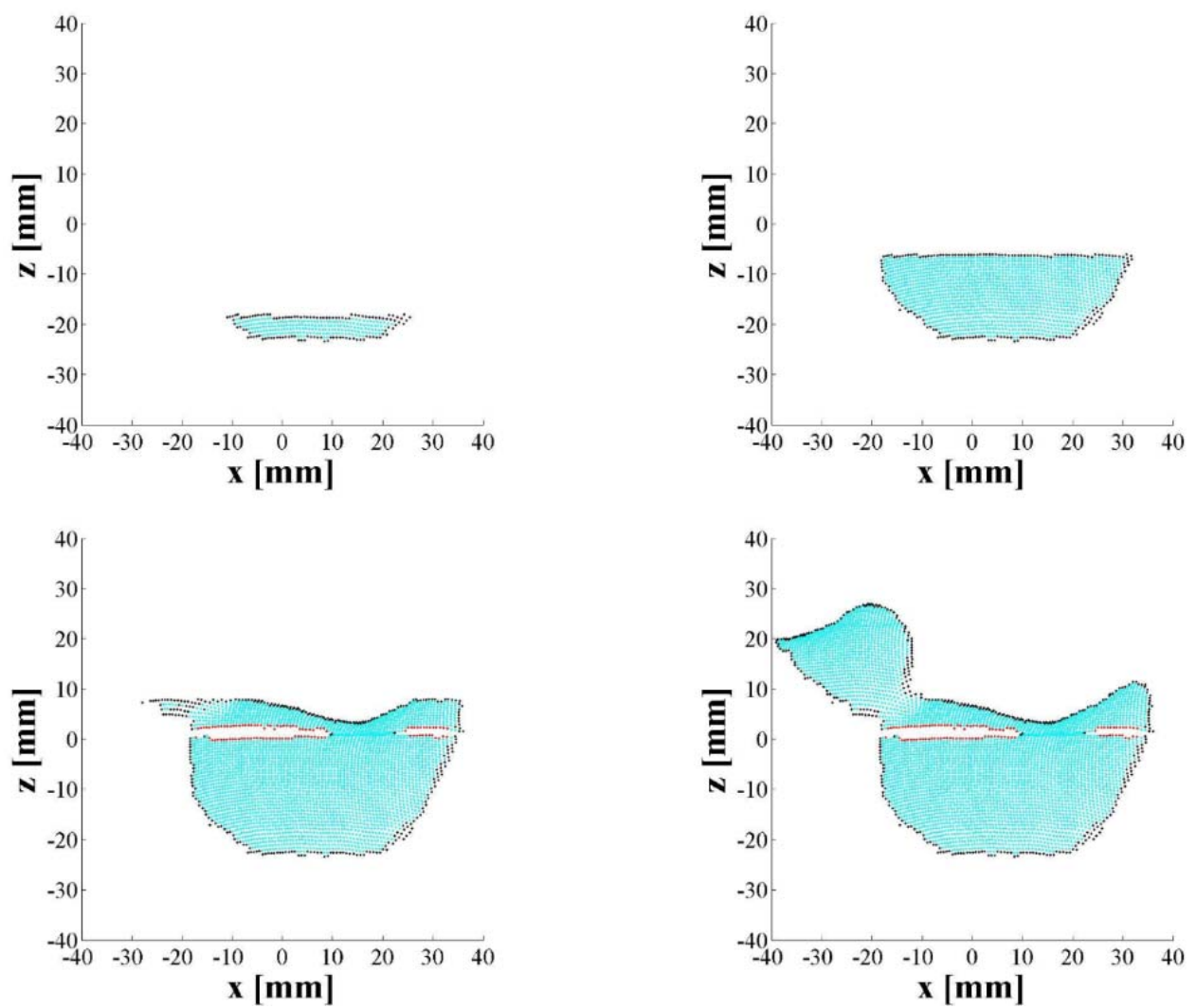

Notes: Points on duck surface of the duck are plotted in cyan; black points are the boundary points; red dots represent the risky points. 
In Figure 10 some results for the comparison between real and expected geometries are shown. Beside the hole, in some areas the distance between the fabricated surface and the expected one is greater than $2 \mathrm{~mm}$. Even in this case, the algorithm is able to generate an alarm and the user can decide whether to interrupt the fabrication process or not.

Figure 10 Results of the simulation for the algorithm to compare the fabricated surface with the expected geometry (see online version for colours)
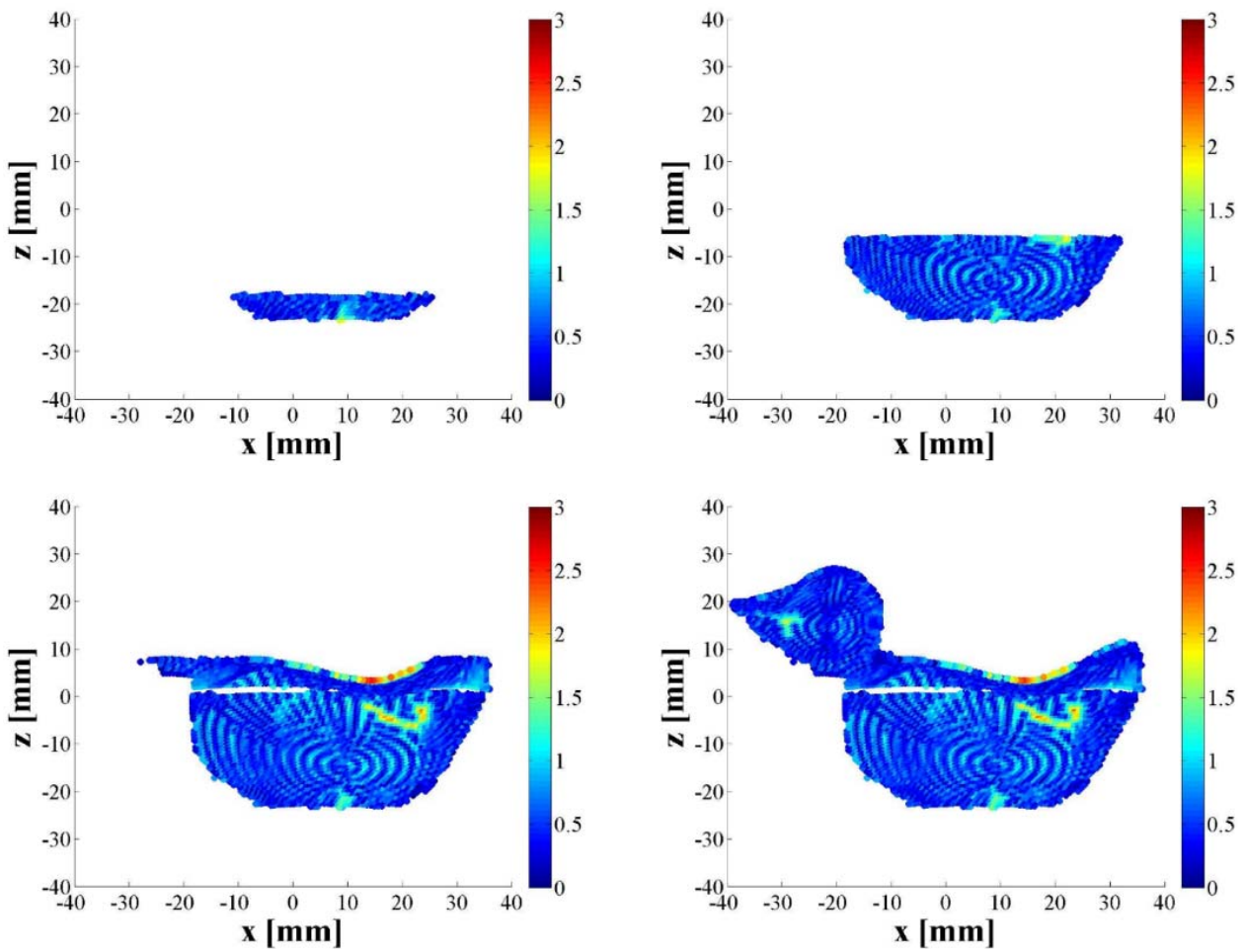

The algorithm has been run on a common laptop with CPU frequency $1.7 \mathrm{GHz}$ : the computational time necessary to analyse the whole dataset (approximately, 4,600 points) with the two algorithms is lower than one minute; since the material deposition rate is much slower, the inspection algorithm can be used to real-time detect the presence of issues, given a suitable class of sensors.

\section{The role of MES}

The technique for surface monitoring can be used as a stand-alone application able to provide alarms in case an issue arises. However, beyond the monitoring and control of the fabrication process, the output of the algorithm can be used with further purposes.

As stated in Section 3, performance and quality monitoring are among the tasks that a MES has to perform. Thus, the efficacy of the monitoring and control system could be enhanced through the integration with a MES. If the monitoring and control system is used offline, MES allows to compare the performance of the last production process with the previous ones and detects symptoms of possible criticalities. The information 
analysed and stored by the MES is an instrument to support automatic correction or compensation strategies, as well as to improve the awareness of operators' decisions. The availability of data-sources able to perform online measurements would further enhance this capability, allowing to adjust the process in real-time and improve the quality of the part undergoing the printing process. The information provided by the MES, in turn, enables to reduce the sources of waste, with particular concern for defects: the detection of issues allows to stop the production of a part as soon as its quality is believed unsatisfactory: hence, the material waste due to finishing a part that will be rejected is avoided, as well as the time spent in useless processes. Thus, the communication between the monitoring and control system and the MES also supports the path towards lean manufacturing (Womack et al., 1990). It must be highlighted that the whole cloud of points is transmitted to the MES, but only a few amount of synthetic indicators is necessary to be saved for each part (e.g., characteristic sizes, maximum/average deviation from the nominal geometry, detected issues).

Over longer time-scales, syntheses of the collected data can be performed to highlight the reasons for which criticalities occurred. This capability is very significant in the field of AM: currently, the most important challenges for this technology are poor part accuracy and lack of process repeatability (Rao et al., 2015). These issues are due to the complex relationships among variables which are, in many cases, still unknown. Hence, the information provided by the MES allows to acquire knowledge about the process and to improve its performance. The feedback mechanism allows to reduce the ramp-up phase of a new product, since machine parameters can be tuned in real-time. Further, the acquired expertise can be used for further product developments, and improved quality of the output can be reached (e.g., lower surface roughness and stair-stepping effect, or improved stress resistance).

\section{Cooperation between MES and DFAM: proposal of a new framework}

A broader deployment of these results can be done if the MES is integrated with tools for design: DFAM has been introduced in Section 2.2.

MES can profitably be supported by design tools: the cooperation between the two systems allows MES to continuously compare the 'as-is' product and process states to the expected conditions and quickly detect mismatchings. In the presented case study, this task is performed by the algorithm that evaluates the adherence of the measured points cloud to the expected output. This cooperation supports the creation of a product passport, which is "a set of information about the components and materials that a product contains, and how they can be disassembled and recycled at the end of the product's useful life" (European Commission, 2013). The improved knowledge about the real condition of the product enables a more realistic definition of its use and its useful life: this thesis has been supported by Portillo-Barco and Charnley (2015), who aim at using sensors to gather data concerning the deployment condition of high pressure nozzle guide vanes and better address the prediction of performance and maintenance.

MES can also profitably support design: the analyses performed over longer time-scales, based on real data, the information about criticalities and the in-field acquired knowledge are a critical input to correct and improve the design of a product or its fabrication process (e.g., change machine parameters, the positioning of the part on the machine tray, change material, choose a different machine, ...). 
Figure 11 Proposed framework for the cooperation between MES and DFAM (see online version for colours)

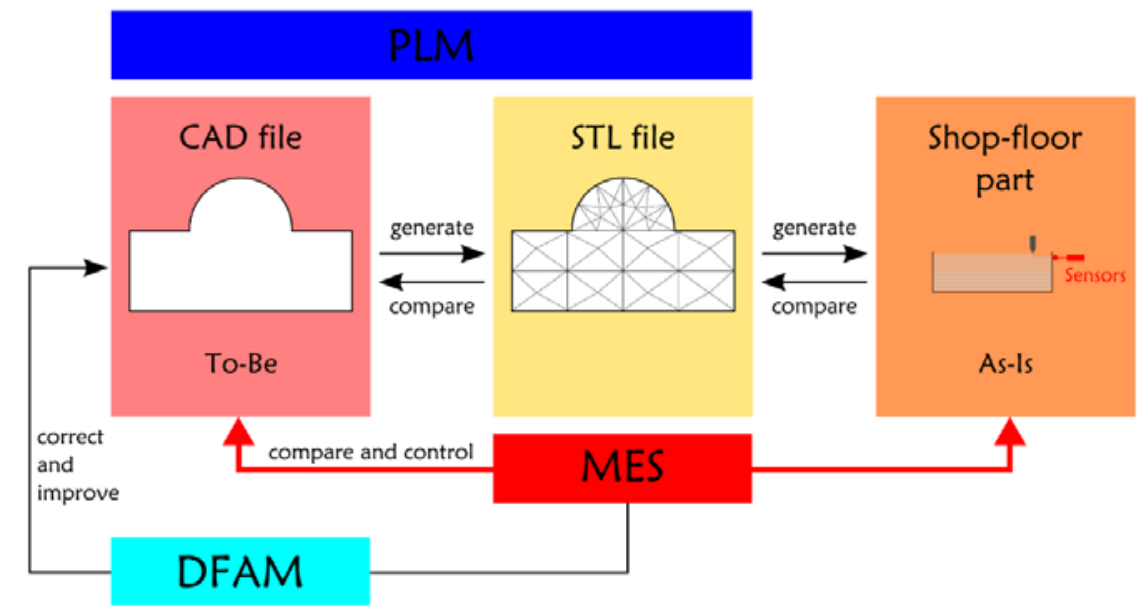

In Figure 11 the framework for the cooperation between the two systems is schematically shown. It allows to extend the DFAM model proposed by Ponche et al. (2014), shown in Figure 5: it consists in three tasks and is able to optimise the design of a product taking into account the capabilities and the constraints (such as the thermal distribution and the trajectories) of the process that will be used for its fabrication. MES support can be useful to improve the result of all the three tasks performed by the DFAM.

First, part orientation has a strong impact on the quality of the finished part. Decision support tools have been developed to identify the part orientation that results in the best roughness and accuracy of the produced object. However, such tools are based on predictions and simulations of process behaviour. A feedback information from a set of sensors able to evaluate the quality of the physical part would be useful to validate the predictions and, in case of mismatching, to correct the orientation of the part into the machine. Also the simulation model can benefit from the feedback mechanism: it can be enriched or adjusted with the empirically acquired knowledge, resulting in a more accurate output; improved models can lead to better predictions and, in turn, to better support the DFAM. Second, the shop-floor information can also be used for further adjustments of the part geometry. In case the quality or the precision of specific features is not satisfactory, the shape of the object and the material distribution can be revised more quickly. The third task included in the DFAM methodology is the optimisation of manufacturing paths and machine parameters. A feedback information from the shop-floor is useful because even minute variations can lead to strong differences in the quality of the fabricated part. Several variables can affect the production process, concerning the deposition and the material (melt pool geometry, temperature, deposition height); furthermore, the output quality is also correlated to the state of the deposition chamber, such as temperature, humidity or oxygen concentration (Reutzel and Nassar, 2015). All these parameters interact with each other. It is not trivial to ensure the quality of a produced part by controlling only a few variables, but further adjustments to the machine parameters can be necessary according to the real operating conditions. Such adjustments can be taken according to the measurements performed by the sensors, and a 
MES-DFAM cooperation allows to quicker take decisions and actions to improve output quality.

This proposal for MES-DFAM cooperation also allows to extend the model proposed by Rosen (2007) (see Figure 4): after planning a manufacturing process, simulations are performed to check whether the design process results in producing an object in compliance with the specifications. Such simulations can be supported by the MES feedback, which allows to continuously validate the process and its model and to better take into account process variability. In case an issue arises, alarms may be generated, or strategies for self-adaptation or self-compensation can be undertaken. Furthermore, functionalities to early detect possible decays of the process over a longer time scale can be implemented.

\section{Conclusions and future work}

In this paper, the importance of deploying a monitoring system in an AM machine has been shown. The results obtained with a test performed offline are promising, and an online implementation would provide useful hints to correct the process while it is still occurring. The scanning system used in this work cannot be deployed in a production environment, since the ratio between costs and benefits would be excessively high. However, several different optical devices can be used to acquire a 3D cloud of points; there exist reliable, recently developed tools which cost is sufficiently low to allow the deployment of several devices to have a complete scan of the object undergoing fabrication (such as the Asus Xtion). Nonetheless, such devices cannot be integrated into the building chamber of an additive machine; a further miniaturisation effort is necessary.

The framework for direct communication between MES and DFAM has also been shown: the two systems can support with each other to improve the control of the process and to enhance the design of a part. This integration enables to use experience-gathered knowledge to address a dynamical change of the way to work, leading to more flexible, efficient and smart processes. Today, this is one of the major trends in manufacturing (Jardim-Goncalves et al., 2016).

Beside these results, the deployment of a MES in the field of additive processes also allows to improve the economical sustainability of the process. Today, due to the unpredictability of the process, the unitary cost of a part produced through AM is usually higher than producing it by the traditional technology. Nonetheless, AM is convenient to produce single parts or small lots, because it does not require the initial investments necessary to start the process in traditional manufacturing (e.g., specialised machines, moulds, ...). However, improved knowledge process results in improved predictability and output quality. This, in turn, can change the economic balance between traditional and AM, and make AM convenient even for larger batch sizes. Further, sensors can be used to monitor parameters linked to the energy impact of the process, such as the nozzle temperature and the energy requested to run the process. Such data can be analysed by the MES to evaluate the current energy efficiency and identify optimisation paths to improve sustainability. A methodology towards this direction has been proposed by Peruzzini et al. (2014).

The study presented in this paper has been applied to a case study in the field of fused deposition modelling. Nevertheless, the approach is general enough to be deployed with any of the available additive technologies discussed in Section 2: the technique presented 
in Section 4.2 needs a cloud of point as input, without regard for the specific technology chosen to produce the part. The deployment of MES could be much more significant in high value-added productions, such as the production of aeronautical or biomechanical components, where the tolerances are very tight and expensive materials are deployed. MES-DFAM cooperation can also be helpful in testing new materials or alloys: the sensors-based system can collect information about the behaviour of the process and the final quality of the product; the acquired data may validate the expected performance, or provide hints for further adjustments or improvements.

\section{References}

Abellan-Nebot, J.V. and Subiron, F.R. (2010) 'A review of machining monitoring systems based on artificial intelligence process models', International Journal of Advanced Manufacturing Technology, Vol. 47, No. 1, pp.237-257.

Arica, E. and Powell, D. (2014) 'A framework for ICT-enabled real-time production planning and control', Advances in Manufacturing, Vol. 2, No. 2, pp.158-164.

ASTM (F2792-12a) (2012) Standard Technology for Additive Manufacturing Technologies, ASTM International, West Conschohocken.

Atzeni, E. and Salmi, A. (2012) 'Economics of additive manufacturing for end-usable metalparts', International Journal of Advanced Manufacturing Technology, Vol. 62, Nos. 9-12, pp.1147-1155.

Basiliere, P. and Shanler, M. (2014) Hype Cycle for 3D Printing [online] $\mathrm{http}: / /$ www.gartner.com/newsroom/id/2825417 (accessed 15 June 2016).

Besl, P.J. and McKay, N.D. (1992) 'Method for registration of 3-D shape', IEEE Transactions on Pattern Analysis, Vol. 14, No. 2, pp.239-256.

Boothroyd, G., Dewhurst, P. and Knight, W. (1994) Product Design for Manufacture and Assembly, CRC Press, Boca Raton, Florida, USA, ISBN: 9781420089271.

Boschetto, A. and Bottini, L. (2014) 'Accuracy prediction in fused deposition modeling', International Journal of Advanced Manufacturing Technology, Vol. 73, Nos. 5-8, pp.913-928.

Bukkapatnam, S. and Clark, B. (2006) 'Dynamic modeling and monitoring of contour crafting - an extrusion-based layered manufacturing process', Journal of Manufacturing Science and Engineering, Vol. 129, No. 1, pp.135-142.

Byun, H.S. and Lee, K.H. (2006) 'Determination of the optimal build direction for different rapid prototyping processes using multi-criterion decision making', Robotics and Computer-Integrated Manufacturing, Vol. 22, No. 1, pp.69-pp.80.

Doubrovski, Z., Verlinden, J.C. and Geraedts, J.M.P. (2011) 'Optimal design for additive manufacturing: opportunities and challenges', Proceedings of the 16th Design for Manufacturing and the Life Cycle Conference, Vol. 9, pp.635-646.

European Commission (2013) European Resource Efficiency Platform Pushes for Product Passports [online] https://ec.europa.eu/environment/ecoap/about-eco-innovation/policiesmatters/eu/20130708_european-resource-efficiency-platform-pushes-for-product-passports_en (accessed 15 June 2016).

Faes, M., Abbeloos, W., Vogeler, F., Valkenaers, H., Coppens, K., Goedemé, T. and Ferraris, E. (2014) 'Process monitoring of extrusion based 3D printing via laser scanning', Proceedings of the 6th International Conference on Polymers and Moulds Innovations(PMI), Vol. 6, pp.363-367.

Huang, Y., Leu, M.C., Mazumder, J. and Donmez, A. (2015) 'Additive manufacturing: current state, future potential, gaps and needs, and recommendations', Journal of Manufacturing Science and Engineering, Vol. 137, No. 1, pp.014001:1-10. 
Jardim-Goncalves, R., Grilo, A. and Popplewell, K. (2016) 'Novel strategies for global manufacturing systems interoperability', Journal of Intelligent Manufacturing, Vol. 27, No. 1, pp.1-9.

Laverne, F., Segonds, F., Anwer, N. and Le Coq, M. (2015) 'Assembly based methods to support product innovation in design for additive manufacturing: an exploratory case study', Journal of Mechanical Design, Vol. 137, No. 12, pp.121701:1-8.

Mançanares, C.G., Zancul, E.D.S., Cavalcante da Silva, J. and Cauchick Miguel, P.A. (2015) 'Additive manufacturing process selection based on parts' selection criteria', International Journal of Advanced Manufacturing Technology, Vol. 80, No. 5, pp.1007-1014.

Mellor, S., Hao, L. and Zhang, D. (2014) 'Additive manufacturing: a framework for implementation', International Journal of Production Economics, Vol. 149, pp.194-201.

Meyer, H., Fuchs, F. and Thiesl, K. (2009) Manufacturing Execution Systems (MES): Optimal Design, Planning, and Deployment, McGraw-Hill Professional, New York, USA.

National Institute of Standards and Technology (NIST) (2013) Measurement Science Roadmap for Metal-Based Additive Manufacturing [online] http://www.nist.gov/el/isd/upload/NISTAdd Mfg_Report_FINAL-2.pdf (accessed 15 June2016).

Perez, A.A., Haid, C.M., Doll, M.P. and Pieper, F.W. (2015) Automatic Process Control of Additive Manufacturing Device, United States Patent No. US2015/0045928A1.

Perret, H. and Philippi, J. (2014) Method for Automatic Calibration of a Device for Generative Production of a Three-Dimensional Object, German Patent No.WO2014/180971A1.

Peruzzini, M., Luzi, A. and Marilungo, E. (2014) 'Assessing sustainability and supporting compliance to standards in continuous industrial processes', International Journal of Product Lifecycle Management, Vol. 7, Nos. 2-3, pp.137-165.

Ponche, R., Hascoet, J.Y., Kerbrat, O. and Mognol, P. (2012) 'A new global approach to design for additive manufacturing', Virtual and Physical Prototyping, Vol. 7, No. 2, pp.93-105.

Ponche, R., Kerbrat, O., Mognol, P. and Hascoet, J.Y. (2014) 'A novel methodology of design for additive manufacturing applied to additive laser manufacturing process', Robotics and Computer-Integrated Manufacturing, Vol. 30, No. 4, pp.389-398.

Portillo-Barco, C. and Charnley, F. (2015) 'Data requirements and assessment of technologies enabling a product passport within products exposed to harsh environments: a case study of a high pressure nozzle guide vane', International Journal of Product Lifecycle Management, Vol. 8, No. 3, pp.253-282.

Rao, P., Liu, J., Roberson, D., Kong, Z.J. and Williams, C. (2015) 'Online real-time quality monitoring in additive manufacturing processes using heterogeneous sensors', Journal of Manufacturing Science and Engineering, Vol. 137, No. 6, pp.061007:1-12.

Reutzel, E.W. and Nassar, A.R. (2015) 'A survey of sensing and control systems for machine and process monitoring of directed-energy, metal-based additive manufacturing', Rapid Prototyping Journal, Vol. 21, No. 2, pp.159-167.

Rosen, D.W. (2007) 'Computer-aided design for additive manufacturing of cellular structures', Computer-Aided Design and Applications, Vol. 4, No. 5, pp.585-594.

Snatkin, A., Karjust, K., Majak, J., Aruväli, T. and Eiskop, T. (2013) 'Real time production monitoring system in SME', Estonian Journal of Engineering, Vol. 19, No. 4, pp.62-75.

Sood, A.K., Equbal, A., Toppo, V., Ohdar, R.K. and Mahapatra, S.S. (2012a) 'An investigation on sliding wear of FDM built parts', CIRP Journal of Manufacturing Science and Technology, Vol. 5, No. 1, pp.48-54.

Sood, A.K., Ohdar, R.K. and Mahapatra, S.S. (2012b) 'Experimental investigation and empirical modelling of FDM process for compressive strength improvement', Journal of Advanced Research, Vol. 3, No. 1, pp.81-90.

Tumbleston, J.R., Shirvanyants, D., Ermoshkin, N. et al. (2015) 'Continuous liquid interface production of 3D objects', Science, Vol. 347, No. 6228, pp.1349-1352.

Vayre, B., Vignat, F. and Villeneuve, F. (2012) 'Metallic additive manufacturing: state-of-the-art review and prospects', Mechanics and Industry, Vol. 13, No. 2, pp.89-96. 
Vijayaraghavan, V., Garg, A., Lam, J., Panda, B. and Mahapatra, S.S. (2014) 'Process characterisation of 3D-printed FDM components using improved evolutionary computational approach', International Journal of Advanced Manufacturing Technology, Vol. 78, Nos. 5-8, pp.781-793.

Wohlers Associates (2014) 3D Printing and Additive Manufacturing State of the Industry [online] http://www.wohlersassociates.com/state-of-the-industry-reports.html (accessed 15 June 2016).

Womack, J.P., Jones, D.T. and Roos, D. (1990) The Machine that Changed the World, Free Press, New York, USA.

Zhang, Y., Bernard, A., Harik, R. and Karunakaran, K.P. (2015) 'Build orientation optimization for multi-part production in additive manufacturing', Journal of Intelligent Manufacturing, in press.

Zhong, R.Y., Dai, Q.Y., Qu, T., Hu, G.J. and Huang, G.Q. (2013) 'RFID-enabled real-time manufacturing execution system for mass-customization production', Robotics and Computer-Integrated Manufacturing, Vol. 29, No. 2, pp.283-292. 